ISSN 1392-3196 / e-ISSN 2335-8947

Zemdirbyste-Agriculture, vol. 107, No. 3 (2020), p. 255-262

DOI $10.13080 /$ z-a.2020.107.033

\title{
Effect of fungal isolates and imidacloprid on cabbage aphid Brevicoryne brassicae and its parasitoid Diaeretiella rapae
}

\author{
Samaneh AKBARI, Shahram MIRFAKHRAIE, Shahram ARAMIDEH, \\ Mohammad Hassan SAFARALIZADEH \\ Urmia University, Faculty of Agriculture Science \\ Urmia, Iran \\ E-mail: sh.aramideh@urmia.ac.ir, sh_mirfakhraie@yahoo.com
}

\begin{abstract}
Diaeretiella rapae (McIntosh) is a primary parasite of the cabbage aphid Brevicoryne brassicae (L.); both species are widely distributed throughout the world. In this research, the efficacy of imidacloprid and five entomopathogenic fungi: Acremonium sclerotigenum, Beauveria bassiana, Paecilomyces variotii, Simplicillium sp. and Lecanicillium muscarium, against cabbage aphid and its parasitoid was evaluated. Concentration-mortality and time-mortality data were subjected to Probit analyses to estimate $\mathrm{LC}_{50}$ (lethal concentration) and $\mathrm{LT}_{50}$ (lethal time) values for each isolate and imidacloprid for adults of $B$. brassicae and D. rapae. The $\mathrm{LC}_{50}$ values for A. sclerotigenum, B. bassiana, P. variotii, Simplicillium sp., L. muscarium and imidacloprid were $2.7 \times 10^{3}, 3.7 \times 10^{3}, 8.1 \times 10^{6}, 4.7 \times 10^{5}$ and 2.5 $\times 10^{3}$ conidia $\mathrm{ml}^{-1}$ and $13.56 \mathrm{ppm}$, respectively. Cumulative mortality of B. brassicae and D. rapae 7 days after treatments was $98.56 \%$ and $99.99 \%$ by $L$. muscarium at high concentration $\left(10^{8}\right.$ conidia $\left.\mathrm{ml}^{-1}\right)$, respectively. The highest mortality of $B$. brassicae and D. rapae after 48 and 72 hours was observed in combinations of sublethal mixed treatments: $\mathrm{LC}_{25}$ (imidacloprid + A . sclerotigenum), $\mathrm{LC}_{25}$ (imidacloprid + B. bassiana), $\mathrm{LC}_{25}$ (imidacloprid + L. muscarium), and $\mathrm{LC}_{25}$ (imidacloprid + A. sclerotigenum), $\mathrm{LC}_{25}$ (imidacloprid + B. bassiana), $\mathrm{LC}_{25}$ (imidacloprid + P. variotii), $\mathrm{LC}_{25}$ (imidacloprid + Simplicillium $\mathrm{sp}$.), $\mathrm{LC}_{25}$ (imidacloprid + L. muscarium), respectively. The $\mathrm{LT}_{50}$ values for $B$. brassicae and $D$. rapae at concentration $10^{8}$ conidia $\mathrm{ml}^{-1}$ were obtained 0.26 and 2.16 days by A. sclerotigenum, and 7.4 and 6.22 days - by P. variotii isolate, respectively. This study showed that imidacloprid had harmful effect on the pest and parasitoid; however, fungal isolates were safer than imidacloprid to the parasitoid. The results of this study show that entomopathogenic fungi can be effectively used alone and mixed with sublethal concentration $\left(\mathrm{LC}_{25}=9.23 \mathrm{ppm}\right)$ of imidacloprid in integrated pest management of $B$. brassicae.
\end{abstract}

Key words: biocontrol, entomopathogenic fungi, parasitoid, pathogenicity, sublethal.

\section{Introduction}

The cabbage aphid, Brevicoryne brassicae L. (Hem.: Aphididae) is a serious pest of cabbage that regularly causes significant yield reductions in brassica crops (Collier, Finch, 2017). Chemical compounds have been vastly used to control $B$. brassicae, which caused development of resistance, destruction of natural enemies and environmental pollution (Pereira et al., 2018). Therefore, considerable effort has been devoted to researching environmentally safe methods for management of $B$. brassicae, particularly biological control (Brennan, 2016; Song et al., 2017). The hymenopteran parasitoid, Diaeretiella rapae (McIntosh) (Hym.: Braconidae, Aphidiinae) is one of the most important naturallyoccurring parasitoids of B. brassicae (Saleh et al., 2009; Singh, Singh, 2015) and is often used in augmentative biological control programs (Neuville et al., 2015).

Among microbial control agents, entomopathogenic fungi have considerable potential to suppress various arthropod pest populations (Güçlü et al., 2010; Sandhu et al., 2012). These fungi present a potential alternative to pesticides that can provide longlasting pest control without undesirable impacts on nontarget organisms or the broader environment (Kim et al., 2006; Jones et al., 2009). However, the best candidates for use in an integrated pest management (IPM) program would be those that are virulent to the target while being innocuous to the pest's predators and parasitoids. Entomopathogenic fungi such as Beauveria bassiana (Balsamo) Vuillemin and Paecilomyces ssp. showed a promising level of activity against aphids (Gurulingappa et al., 2011; Sandhu et al., 2012). Rashki et al. (2009) demonstrated that entomopathogenic fungi $B$. bassiana had no adverse effect on biological parameters of the parasitoid wasp, Aphidius matricariae (Hymenoptera: Braconidae) and can be successfully combined for biological control of Myzus persicae (Hemiptera:

Please use the following format when citing the article:

Akbari S., Mirfakhraie Sh., Aramideh Sh., Safaralizadeh M. H. 2020. Effect of fungal isolates and imidacloprid on cabbage aphid Brevicoryne brassicae and its parasitoid Diaeretiella rapae. Zemdirbyste-Agriculture, 107 (3): $255-262$. DOI 10.13080/z-a.2020.107.033 
Aphididae). B. bassiana and Paecilomyces fumosoroseus (Wize) Brown and Smith have been reported as most important entomopathogenic fungi (Al-Olayan, 2013).

The Lecanicillium lecanii has been reported to cause natural epizootics in aphid and mealy bug populations in natural conditions (Shah, Pell, 2003). The L. lecanii isolates were more infective compared to other entomopathogenic fungi in order Hypocreales (Ascomycota) as well as B. bassiana, Paecilomyces sp. and Metarhizium anisopliae against aphid species (Loureiro, Moino, 2006). Additionally, imidacloprid, a neonicotinoid insecticide, is a contact and oral chemical which binds selectively to the nicotinic acetylcholine receptor to control sucking insects such as cabbage aphids (Roessink et al., 2013).

In IPM programs, it is essential to know the influence of the compatibility between entomopathogenic fungi and pesticide used in crop protection (Sain et al., 2019). Therefore, use of entomopathogenic fungi as biopesticides in combination with sublethal dose of chemical agents should be considered. Moreover, Rashki et al. (2015) reported that sublethal dose of imidacloprid causes restlessness in aphids and enhances the likelihood of development of entomopathogenic conidia from their contaminated habitat. Thus, additional information is needed to get better understanding of the impacts of pesticides and entomopathogenic fungi on natural enemies (Kim et al., 2006; Jones et al., 2009). Entomopathogenic fungi can be used in combination with the parasitoids and predators as biological control agents for controlling the aphids. This method requires an effective time management in order to prevent the antagonistic interactions (Seiedy et al., 2015).

The aims of this study were (1) to determine susceptibility of Brevicoryne brassicae and its parasitoid Diaeretiella rapae to five entomopathogenic fungi: Acremonium sclerotigenum, Beauveria bassiana, Paecilomyces variotii, Simplicillium sp., Lecanicillium muscarium, and imidacloprid as insecticide with $\mathrm{LC}_{50}$ and $\mathrm{LT}_{50}$ values, and (2) estimate their combination effect for use in integrated aphid management with the least effect on parasitoid.

\section{Materials and methods}

This research was performed in a greenhouse at the Agriculture Faculty of Urmia University, Iran during 2018 under controlled conditions of $25 \pm 2{ }^{\circ} \mathrm{C}$ temperature, $60 \pm 5 \%$ relative air humidity and $16 / 8$ (light/dark) photoperiod.

Rearing of Brassicae oleracea. Cabbages were grown from seed in plastic flower pots $(20.0 \mathrm{~cm}$ diameter $\times 23.0 \mathrm{~cm}$ height), filled with a potting soil mix compost and peat moss, fertilized once with $\mathrm{N}_{15} \mathrm{P}_{15} \mathrm{~K}_{15}$ and watered as needed.

Rearing of Brevicoryne brassicae. Cabbage aphids $B$. brassicae (were collected from Brassicaceae plants in the region of Nazlo village $\left(37.5287^{\circ} \mathrm{N}, 45.0469^{\circ}\right.$ E) near Urmia city, Iran. Aphids were reared on common cabbage ( $B$. oleracea var. capitate) at a six-leaf stage in $500 \mathrm{ml}$ pots in wooden framed cages $(45 \times 50 \times 70 \mathrm{~cm})$ covered by cheesecloth and maintained in a greenhouse. Adult females from the stock colonies were used to obtain nymphs and new adults for the experiments.

Rearing of Diaeretiella rapae. A colony of $D$. rapae was reared on $B$. brassicae under the same greenhouse conditions. Aphid mummies were collected from cabbage fields by cutting infected leaves and kept until emergence in net cover cages $(90 \times 90 \times 70 \mathrm{~cm})$. All emerged parasitoides were fed a $30 \%$ honey solution and used to parasitize aphids or in bioassay experiments, $24 \mathrm{~h}$ later.

Parasitism test. For parasitism, a total of 250 forth nymph aphids were allowed to settle on a freshly excised leaf of cabbage $(4.0 \mathrm{~cm}$ diameter $)$ placed in a plastic Petri dish $(9.0 \mathrm{~cm}$ diameter $)$ by a fine paint brush on moistened filter paper. Therefore, fifteen mated females of $D$. rapae that emerged were gently introduced by an aspirator into Petri dishes ( 5 in each dish) on 250 forth nymph aphids ( 25 in each dish) for $24 \mathrm{~h}$. After one day, the parasitoids were removed and the hosts were left undisturbed until they mummified. After mummification, they were collected and transferred to sterilized Petri dishes $(9.0 \mathrm{~cm}$ diameter) for emergence and bioassay test. The experiment was replicated three times.

Preparation of fungal isolates and conidial suspensions. Five fungal isolates: Acremonium sclerotigenum FCCUU490, Beauveria bassiana FCCUU438, Paecilomyces variotii, Simplicillium sp. FCCUU478 and Lecanicillium muscarium FCCUU420, were obtained from the collection of the Plant Protection Department of Urmia University and cultured on SDA (Sabouraud dextrose agar) at $25 \pm 1^{\circ} \mathrm{C}$ temperature for two weeks to induce sporulation. Spores were removed from the surface of media with a sterile scalpel and transferred to a test tube containing sterilized distilled water with $0.05 \%$ Tween 80 as surfactant. After the tubes were shaken, the spore suspension was filtered through three layers of muslin cloth to remove hyphal debris. Spores were counted with a Neubauer hemocytometer (LABART, Germany) and serial dilutions were made to obtain conidial concentrations of $10^{4}, 10^{5}, 10^{6}, 10^{7}$ and $10^{8}$ conidia $\mathrm{ml}^{-1}$.

Insecticide. Commercial formulations of imidacloprid $350 \mathrm{~g} \mathrm{~L}^{-1}$ Confidor SC (Bayer CropScience, www.cropscience.bayer.com) suspension of $0.35 \%$ concentration were used in the bioassay test.

Concentration-mortality (LC) and timemortality (LT) values of aphid and parasitoid to fungi. Bioassay test was carried out on aphid and its parasitoid. Different concentrations $\left(10^{4}\right.$ to $10^{8}$ conidia $\left.\mathrm{ml}^{-1}\right)$ of each fungal isolate as main concentrations base of preliminary test were prepared. Aphids were treated by fungal concentrations. The sterilized cabbage leaves with 10 aphids were dipped in each concentration with $0.05 \%$ Tween 80 for 10 seconds then were placed in Petri dishes. The control treatment was dipped in water with $0.05 \%$ Tween 80 . We confirmed that $0.05 \%$ Tween 80 is not harmful to aphids or conidial germination in preexperiments. The mortality was recorded for 7 days. To estimate $\mathrm{LT}_{50}$ value, aphid mortality was recorded every 12 hours. Newly born nymphs were counted and removed daily from the plants. Aphid cadavers were disinfected using $2 \% \mathrm{NaClO}$ (sodium hypochlorite) and rinsed with sterile distilled water. The cadavers were then incubated in a humidity chamber (100\% relative humidity) into a Petri dish on damp filter paper to ensure that death was due to fungal treatment. Only aphids which exhibited fungal sporulation were considered to have died from the fungal treatment. The whole experiment was conducted with three replicates (Fadayivata et al., 2014). 
LC and LT values of aphid and parasitoid to imidacloprid. In order to determine $\mathrm{LC}_{50}$ value of imidacloprid, first in a series of preliminary tests, the maximum and minimum concentrations were determined with $80-20 \%$ mortality on the adult insect, and then between maximum and minimum, three concentrations were measured by logarithm method (Bayramzadeh et al., 2019). Thus, five concentrations (10, 13.16, 17.32, 22.80 and $30.00 \mathrm{ppm}$ ) with distilled water as control, each concentration in three replicates, were sprayed on cabbage leaves and 10 adult aphids were released in each replication. The mortality of different concentrations after 24, 48 and 72 hours was recorded. To estimate $\mathrm{LT}_{50}$ value, aphid mortality was recorded every 12 hours. Newly born nymphs were counted and removed daily from the plants.

Percentages of emergence of parasitoid from infested aphids by fungal isolates. This experiment was laid out in a completely randomized design with 12 treatments: $\mathrm{LC}_{50}$ A. sclerotigenum, $\mathrm{LC}_{50}$ B. bassiana, $\mathrm{LC}_{50}$ P. variotii, $\mathrm{LC}_{50}$ Simplicillium $\mathrm{sp} ., \mathrm{LC}_{50}$ L. muscarium, $\mathrm{LC}_{50}^{50}$ imidacloprid, $\mathrm{LC}_{25}$ (imidacloprid $+A$. sclerotigenum), $\mathrm{LC}_{25}$ (imidacloprid $+B$. bassiana), $\mathrm{LC}_{25}$ (imidacloprid $+P$. variotii), $\mathrm{LC}_{25}$ (imidacloprid + Simplicillium $\mathrm{sp}$.), $\mathrm{LC}_{25}$ (imidacloprid + L. muscarium) and distilled water as control sprayed in three replications on 150 mummy aphids. B. brassica mummies were collected from the aphid rearing cage and 50 mummy aphids were placed in each Petri dish ( $60 \mathrm{~mm}$ diameter) on leaves of cabbage in the cage. The cages were incubated in a climate chamber at $25 \pm 2{ }^{\circ} \mathrm{C}$ temperature, $60 \pm 5 \%$ relative air humidity and $16 / 8 \mathrm{~h}$ light/dark regime until the parasitoids emerged (48 h). Then mortality of mummy was recorded.

Lethal effect of fungi and imidacloprid combination. After calculating $\mathrm{LC}_{50}$ and $\mathrm{LC}_{25}$ values for fungal isolates and imidacloprid on adult insects, combination effects of fungi and pesticide were evaluated in the laboratory. Therefore, in the preliminary test to detect pesticide side effects of five entomopathogenic fungi on conidia viability were evaluated using a method modified by Lazreg et al. (2009). A conidial suspension was adjusted to $1 \times 10^{4}$ conidia $\mathrm{ml}^{-1}$, and $0.1 \mathrm{ml}^{-1}$ was sprayed on to 6 -cm diameter Petri dishes containing SDA. Petri dishes were maintained at $25 \pm$ $2^{\circ} \mathrm{C}$ temperature. After $24 \mathrm{~h}$ of incubation, percentages of germinated conidia were calculated. Conidia were regarded as germinated when they produced a germ tube at least half of the conidial length. Germination ratios for each fungus were calculated after examining a minimum of 200 conidia from each of three replicate plates. All experiments were conducted in a completely randomized design in 12 treatments, including $\mathrm{LC}_{50}$ A. sclerotigenum, $\mathrm{LC}_{50}$ B. bassiana, $\mathrm{LC}_{50}$ P. variotii, $\mathrm{LC}_{50}$ Simplicillium $\mathrm{sp}$., $\mathrm{LC}_{50}^{50}$ L. muscarium, $\mathrm{LC}_{50}^{50}$ imidacloprid, $\mathrm{LC}_{25}$ (imidacloprid $+A$. sclerotigenum), $\mathrm{LC}_{25}$ (imidacloprid $+B$. bassiana), $\mathrm{LC}_{25}$ (imidacloprid $+P$. variotii), $\mathrm{LC}_{25}$ (imidacloprid + Simplicillium sp.), $\mathrm{LC}_{25}$ (imidacloprid + L. muscarium) and distilled water as control in three replications on ten aphids and its parasitoid. The mortality after 48 and 72 hours was calculated. The percentage of hatching parasitoid in all treatments was calculated as mortality at the same time.

Statistical analysis. The $\mathrm{LC}_{25}, \mathrm{LC}_{50}$ and $\mathrm{LT}_{50}$ values (with $95 \%$ confidence limits) were calculated using the Probit analysis method after correcting according to Abbott's (1925) formula. All data were tested for normality and homogeneity of variance by the ShapiroWilk and Levene's test, respectively. The data collected were subjected to analysis of variance (ANOVA) and means were separated using Turkey's HSD (honestly significant difference) test at a significance level of $P \leq$ 0.05 with software package SPSS, version 22.0 (IBM Inc., USA).

\section{Results}

Determination of concentration-mortality $\left(L C_{50}\right)$ value response. Conidia viability was assessed before each bioassay. Almost 96, 97, 96, 95 and $98 \%$ of conidia of five fungal isolates Acremonium sclerotigenum, Beauveria bassiana, Paecilomyces variotii, Simplicillium sp. and Lecanicillium muscarium were germinated, respectively. The $\mathrm{LC}_{50}$ values of fungal isolates: $A$. sclerotigenum, B. bassiana, $P$. variotii, Simplicillium sp., L. muscarium, and imidacloprid against the adult stage of aphids were determined. The highest mortality between the fungi isolates against the adult stage of aphid was observed in L. muscarium with $\mathrm{LC}_{50}$ value equal $2.5 \times$ $10^{3}$ conidia $\mathrm{ml}^{-1}$ (Table 1$)$.

The $\mathrm{LC}_{50}$ values of imidacloprid and fungal isolates: A. sclerotigenum, B. bassiana, P. variotii, Simplicillium sp. and L. muscarium, of Diaeretiella rapae are shown in Table 2. Fungal isolates L. muscarium and $P$. variotii with $\mathrm{LC}_{50}$ values equal to $5.2 \times 10^{3}$ and $4.0 \times$ $10^{7}$ conidia $\mathrm{ml}^{-1}$ had the highest and the lowest effects on D. rapae, respectively.

Table 1. The $\mathrm{LC}_{25}, \mathrm{LC}_{50}$ and $\mathrm{LC}_{95}$ values of fungal isolates (conidia $\mathrm{ml}^{-1}$ ) and imidacloprid (ppm) against the adult stage of Brevicoryne brassicae

\begin{tabular}{|c|c|c|c|c|c|c|c|}
\hline Isolate & Slope \pm SE & $\begin{array}{l}\text { Intercept } \\
\quad+5\end{array}$ & $\chi^{2}(\mathrm{df})$ & $P$ & $\mathrm{LC}_{25}$ (CLs) & $\mathrm{LC}_{50}(\mathrm{CLs})$ & $\mathrm{LC}_{90}(\mathrm{CLs})$ \\
\hline $\begin{array}{l}\text { Acremonium } \\
\text { sclerotigenum }\end{array}$ & $0.59 \pm 0.14$ & 2.973 & $1.053(3)$ & 0.788 & $\begin{array}{c}1.9 \times 10^{2} \\
\left(0.5-1.8 \times 10^{3}\right)\end{array}$ & $\begin{array}{c}2.7 \times 10^{3} \\
\left(7.5 \times 10^{1}-1.2 \times 10^{4}\right)\end{array}$ & $\begin{array}{c}1.6 \times 10^{6} \\
\left(4.3 \times 10^{5}-3.4 \times 10^{7}\right)\end{array}$ \\
\hline $\begin{array}{l}\text { Beauveria } \\
\text { bassiana }\end{array}$ & $0.70 \pm 0.17$ & 2.496 & $1.909(3)$ & 0.592 & $\begin{array}{c}4.1 \times 10^{2} \\
\left(2.4-2.6 \times 10^{3}\right)\end{array}$ & $\begin{array}{c}3.7 \times 10^{3} \\
\left(1.7 \times 10^{2}-1.3 \times 10^{4}\right)\end{array}$ & $\begin{array}{c}8.4 \times 10^{5} \\
\left(2.5 \times 10^{5}-1.5 \times 10^{7}\right)\end{array}$ \\
\hline $\begin{array}{l}\text { Paecilomyces } \\
\text { variotii }\end{array}$ & 0.08 & 2.525 & $0.630(3)$ & 0.889 & $\begin{array}{c}1.1 \times 10^{5} \\
\left(7.02 \times 10^{3}-4.6 \times 10^{5}\right)\end{array}$ & $\begin{array}{c}8.1 \times 10^{6} \\
\left(2.0 \times 10^{6}-7.3 \times 10^{7}\right)\end{array}$ & $\begin{array}{c}3.2 \times 10^{11} \\
\left(6.3 \times 10^{9}-5.8 \times 10^{15}\right)\end{array}$ \\
\hline Simplicillium sp. & $0.21 \pm 0.07$ & 3.781 & $0.049(3)$ & 0.997 & $\begin{array}{c}3.4 \times 10^{2} \\
\left(1.4 \times 10^{2}-1.3 \times 10^{4}\right)\end{array}$ & $\begin{array}{c}4.7 \times 10^{5} \\
\left(1.1 \times 10^{4}-5.8 \times 10^{6}\right)\end{array}$ & $\begin{array}{c}2.1 \times 10^{13} \\
\left(1.8 \times 10^{10}-1.0 \times 10^{29}\right)\end{array}$ \\
\hline $\begin{array}{l}\text { Lecanicillium } \\
\text { muscarium }\end{array}$ & 0.64 & 2.808 & $0.682(3)$ & 0.877 & $\begin{array}{c}2.2 \times 10^{2} \\
\left(0.5-1.9 \times 10^{3}\right)\end{array}$ & $\begin{array}{c}2.5 \times 10^{3} \\
\left(5.9 \times 10^{1}-1.1 \times 10^{4}\right)\end{array}$ & $\begin{array}{c}9.3 \times 10^{5} \\
\left(2.5 \times 10^{5}-1.9 \times 10^{7}\right)\end{array}$ \\
\hline Imidacloprid & $4.04 \pm 0.73$ & 0.422 & $2.561(3)$ & 0.464 & $\begin{array}{c}9.23 \\
(6.57-11.07)\end{array}$ & $\begin{array}{c}13.56 \\
(11.39-15.40)\end{array}$ & $\begin{array}{c}34.62 \\
(27.45-54.72)\end{array}$ \\
\hline
\end{tabular}

$\mathrm{SE}$ - standard error, $\mathrm{df}$ - degree of freedom, CLs - confidence limits 
Table 2. The $\mathrm{LC}_{25}, \mathrm{LC}_{50}$ and $\mathrm{LC}_{95}$ values of fungal isolates (conidia $\mathrm{ml}^{-1}$ ) and imidacloprid (ppm) against the adult stage of Diaeretiella rapae

\begin{tabular}{|c|c|c|c|c|c|c|c|}
\hline Isolate & lope \pm SE & $\begin{array}{l}\text { Intercept } \\
+5\end{array}$ & $\chi^{2}(\mathrm{df})$ & $P$ & $\mathrm{LC}_{25}$ (CLs) & $\mathrm{LC}_{50}(\mathrm{CLs})$ & $\mathrm{LC}_{90}(\mathrm{CLs})$ \\
\hline $\begin{array}{l}\text { Acremoniun } \\
\text { sclerotigen }\end{array}$ & $41+009$ & 3.282 & 3) & 0.933 & $\begin{array}{r}3.2 \\
(1.1-3 .\end{array}$ & $\begin{array}{r}1.3 \\
6.5 \times 10^{2}\end{array}$ & $\begin{array}{r}1.1 \\
\left(1.4 \times 10^{7}\right.\end{array}$ \\
\hline $\begin{array}{l}\text { Beauveria } \\
\text { bassiana }\end{array}$ & & 85 & ) & $0.46>$ & $\begin{array}{c}3.5 \times 10^{3} \\
\left(2.3 \times 10^{2}-1.5 \times 10^{5}\right)\end{array}$ & $\begin{array}{c}6.0 \times 10^{4} \\
\left(1.3 \times 10^{4}-1.6 \times 10^{5}\right)\end{array}$ & $\begin{array}{c}6.2 \times 10^{7} \\
\left(1.2 \times 10^{7}-1.3 \times 10^{9}\right)\end{array}$ \\
\hline $\begin{array}{l}\text { Paecilomyces } \\
\text { variotii }\end{array}$ & 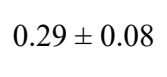 & 992 & & 0 & $\begin{array}{c}1.9 \times 10^{5} \\
\left(6.3 \times 10^{3}-1.06 \times 10^{6}\right)\end{array}$ & $\begin{array}{r}4.0 \\
(7.2 \times 10\end{array}$ & $\begin{array}{r}1.8 \\
\left(5.3 \times 10^{10}\right.\end{array}$ \\
\hline 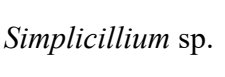 & 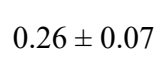 & 3.601 & 0. & 0.902 & $\begin{array}{r}5.2 \times \\
(0.2-1.7\end{array}$ & $\begin{array}{r}1.7 \\
(7.2 \times 10\end{array}$ & $\begin{array}{c}2.6 \times 10^{11} \\
\left(2.2 \times 10^{9}-3.8 \times 10^{18}\right)\end{array}$ \\
\hline $\begin{array}{l}\text { Lecanicillium } \\
\text { muscarium }\end{array}$ & 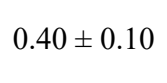 & 3.365 & ) & 0.636 & $\begin{array}{r}1.5 \\
(0.24-1\end{array}$ & $\begin{array}{r}5.2 \\
\left(1.3 \times 10^{2}\right.\end{array}$ & $\begin{array}{c}2.8 \times 10^{7} \\
\left(4.5 \times 10^{6}-2.1 \times 10^{9}\right)\end{array}$ \\
\hline Imidacloprid & $.23 \pm 0.58$ & 1.431 & $3.230(3)$ & 0.702 & $\begin{array}{c}7.82 \\
(3.22-12.5) \\
\end{array}$ & $\begin{array}{c}12.64 \\
(0.15-21.41) \\
\end{array}$ & $\begin{array}{c}31.44 \\
(19.48-606.32) \\
\end{array}$ \\
\hline
\end{tabular}

Explanation under Table 1

Determination of time-mortality ( $\left.\mathrm{LT}_{50}\right)$ value response. The $\mathrm{LT}_{50}$ values of imidacloprid and fungal isolates $A$. sclerotigenum, $B$. bassiana, $P$. variotii, Simplicillium sp. and L. muscarium at $10^{8}$ and $10^{7}$ conidia $\mathrm{ml}^{-1}$ against $B$. brassicae and $D$. rapae are shown in Tables 3 and 4.

The $\mathrm{LT}_{50}$ values of imidacloprid and fungal isolates at two $\left(10^{8}\right.$ and $10^{7}$ conidia $\left.\mathrm{ml}^{-1}\right)$ concentrations showed that $A$. sclerotigenum was the most effective entomopathogenic fungi in control of B. brassicae after imidacloprid (Table 3 ). The $\mathrm{LT}_{50}$ values of imidacloprid and fungal isolates at two $\left(10^{8}\right.$ and $10^{7}$ conidia $\left.\mathrm{ml}^{-1}\right)$ concentrations showed that $L$. muscarium was the most effective entomopathogenic fungi against $D$. rapae after imidacloprid (Table 4).

Comparison of different concentrations of fungal isolates on $B$. brassicae and $D$. rapae. The bioassay results showed that fungal concentrations $\left(10^{8}, 10^{7}, 10^{6}, 10^{5}\right.$ and $10^{4}$ conidia $\left.\mathrm{ml}^{-1}\right)$ against adult $B$. brassicae showed significant differences with $\left(\mathrm{F}_{5,12}=45.78, P=0.001\right),\left(\mathrm{F}_{5,12}=56.98, P=0.001\right)$, $\left(\mathrm{F}_{5,12}^{5,12}=39.69, P=0.001\right),\left(\mathrm{F}_{5,12}{ }^{5}=44.64, P=0.001\right)$ and $\left(\mathrm{F}_{5,12}^{5,12}=21.56, P=0.001\right)$ and on D. rapae $\left(\mathrm{F}_{5,12}=112.80\right.$, $P=0.001),\left(\mathrm{F}_{5,12}=36.21, P=0.001\right),\left(\mathrm{F}_{5,12}^{5,12}=23.27\right.$,

Table 3. The $\mathrm{LT}_{50}$ values of fungal isolates at $10^{8}$ and $10^{7}$ conidia $\mathrm{ml}^{-1}$ and imidacloprid at $\mathrm{LC}_{50}=13.56$ ppm against Brevicoryne brassicae after 7 days

\begin{tabular}{|c|c|c|c|c|c|c|}
\hline $\begin{array}{c}\text { Fungal conidia } \\
\mathrm{ml}^{-1}\end{array}$ & Isolate & $\begin{array}{c}\text { Intercept(a) } \\
+5\end{array}$ & $\chi^{2}$ & $P$ & Slope \pm SE & $\begin{array}{c}\mathrm{LT}_{50} \text { (day) } \\
\text { (CLs) }\end{array}$ \\
\hline \multirow{5}{*}{$10^{8}$} & A. sclerotigenum & 5.76 & 1.52 & 0.91 & $6.32 \pm 0.44$ & $0.26(0.03-0.70)$ \\
\hline & B. bassiana & 3.48 & 5.70 & 0.33 & $5.34 \pm 0.62$ & $1.92(1.66-2.16)$ \\
\hline & P. variotii & 3.41 & 5.31 & 0.37 & $1.81 \pm 0.40$ & $7.40(5.62-13.38)$ \\
\hline & Simplicillium $\mathrm{sp}$. & 3.96 & 1.54 & 0.90 & $2.23 \pm 0.36$ & $2.92(2.33-3.53)$ \\
\hline & L. muscarium & 4.41 & 0.91 & 0.96 & $4.96 \pm 0.75$ & $1.32(1.09-1.52)$ \\
\hline \multirow{5}{*}{$10^{7}$} & A. sclerotigenum & 4.84 & 2.18 & 0.82 & $3.09 \pm 0.37$ & $1.18(0.69-1.59)$ \\
\hline & B. bassiana & 2.88 & 5.24 & 0.38 & $4.21 \pm 0.52$ & $3.19(2.80-3.57)$ \\
\hline & P. variotii & 2.50 & 6.87 & 0.23 & $2.80 \pm 0.56$ & $7.78(6.28-11.76)$ \\
\hline & Simplicillium sp. & 3.29 & 1.23 & 0.94 & $2.73 \pm 0.42$ & $4.27(3.58-5.00)$ \\
\hline & L. muscarium & 3.27 & 0.744 & 0.98 & $2.81 \pm 0.43$ & $4.14(3.54-4.89)$ \\
\hline $13.56 \mathrm{ppm}$ & Imidacloprid & 4.32 & 0.47 & 0.52 & $2.34 \pm 0.71$ & $1.93(1.42-2.77)$ \\
\hline
\end{tabular}

Explanation under Table 1

Table 4. The $\mathrm{LT}_{50}$ values of fungal isolates at $10^{8}$ and $10^{7}$ conidia $\mathrm{ml}^{-1}$ and imidacloprid at $\mathrm{LC}_{50}=12.64$ ppm against Diaeretiella rapae after 7 days

\begin{tabular}{|c|c|c|c|c|c|c|}
\hline $\begin{array}{c}\text { Fungal conidia } \\
\mathrm{ml}^{-1}\end{array}$ & Isolate & $\begin{array}{c}\text { Intercept(a) } \\
+5\end{array}$ & $\chi^{2}$ & $P$ & Slope \pm SE & $\begin{array}{c}\mathrm{LT}_{50} \text { (day) } \\
\text { (CLs) }\end{array}$ \\
\hline \multirow{5}{*}{$10^{8}$} & A. sclerotigenum & 3.46 & 2.06 & 0.84 & $3.15 \pm 0.40$ & $2.16(1.77-2.52)$ \\
\hline & B. bassiana & 3.16 & 11.15 & 0.04 & $3.65 \pm 0.45$ & $3.16(2.29-4.08)$ \\
\hline & P. variotii & 3.68 & 1.92 & 0.86 & $1.65 \pm 0.37$ & $6.22(4.74-10.41)$ \\
\hline & Simplicillium sp. & 3.46 & 4.14 & 0.52 & $2.41 \pm 0.39$ & $4.38(3.67-5.40)$ \\
\hline & L. muscarium & 4.02 & 3.05 & 0.69 & $4.17 \pm 0.52$ & $1.71(1.42-1.97)$ \\
\hline \multirow{5}{*}{$10^{7}$} & A. sclerotigenum & 3.72 & 2.46 & 0.75 & $2.46 \pm 0.37$ & $3.32(2.76-3.96)$ \\
\hline & B. bassiana & 2.84 & 9.44 & 0.093 & $3.31 \pm 0.47$ & $4.46(3.45-6.15)$ \\
\hline & P. variotii & 2.38 & 0.61 & 0.98 & $2.80 \pm 0.63$ & $8.61(6.76-14.85)$ \\
\hline & Simplicillium sp. & 2.79 & 4.62 & 0.46 & $2.69 \pm 0.50$ & $6.59(5.45-9.07)$ \\
\hline & L. muscarium & 3.54 & 4.06 & 0.54 & $3.03 \pm 0.40$ & $3.04(2.57-3.51)$ \\
\hline $12.64 \mathrm{ppm}$ & Imidacloprid & 4.34 & 0.20 & 0.65 & $3.00 \pm 0.73$ & $1.65(1.25-2.06)$ \\
\hline
\end{tabular}

Explanation under Table 1 
$P=0.001),\left(\mathrm{F}_{5,12}=28.02, P=0.001\right)$ and $\left(\mathrm{F}_{5,12}=51.25\right.$, $P=0.001)$ conditions, respectively. The cumulative mortality of aphid and parasitoid caused by L. muscarium, B. bassiana and A. sclerotigenum was 98.6, 97.2, $96.4 \%$ and $99.9,96.5$ and $98.6 \%$, respectively (Table 5).
Combination effects of imidacloprid and fungus on B. brassicae and D. rapae. The effect of different treatments on adult of $B$. brassicae and its parasitoid D. rapae after 48 and 72 hours showed that there were significant differences between the treatments

Table 5. Effect of conidial concentration of fungal isolates against adult of B. brassicae and D. rapae

\begin{tabular}{cccccccc}
\hline \multirow{2}{*}{ Isolate } & \multirow{2}{*}{$\begin{array}{c}\text { Concentration } \\
\left.\text { (conidia m- })^{-1}\right)\end{array}$} & $\begin{array}{c}\text { Lecanicillium } \\
\text { muscarium }\end{array}$ & $\begin{array}{c}\text { Acremonium } \\
\text { sclerotigenum }\end{array}$ & $\begin{array}{c}\text { Beauveria } \\
\text { bassiana }\end{array}$ & $\begin{array}{c}\text { Simplicillium } \\
\text { sp. }\end{array}$ & $\begin{array}{c}\text { Paecilomyces } \\
\text { variotii }\end{array}$ & Control \\
\hline \multirow{3}{*}{$\begin{array}{c}\text { Brevicoryne } \\
\text { brassicae }\end{array}$} & $10^{8}$ & $98.6 \pm 0.01 \mathrm{a}$ & $97.2 \pm 0.01 \mathrm{a}$ & $96.4 \pm 0.01 \mathrm{a}$ & $71.2 \pm 3.32 \mathrm{~b}$ & $63.3 \pm 0.33 \mathrm{~b}$ & $7.3 \pm 0.33 \mathrm{c}$ \\
& $10^{7}$ & $95.7 \pm 0.33 \mathrm{a}$ & $95.3 \pm 0.33 \mathrm{a}$ & $94.7 \pm 0.33 \mathrm{a}$ & $61.0 \pm 0.33 \mathrm{~b}$ & $46.7 \pm 0.88 \mathrm{~b}$ & $12.2 \pm 0.03 \mathrm{c}$ \\
& $10^{6}$ & $93.3 \pm 1.15 \mathrm{a}$ & $86.7 \pm 1.15 \mathrm{a}$ & $94.3 \pm 0.57 \mathrm{a}$ & $63.2 \pm 1.01 \mathrm{~b}$ & $43.3 \pm 0.57 \mathrm{~b}$ & $9.7 \pm 0.03 \mathrm{c}$ \\
& $10^{5}$ & $83.3 \pm 0.33 \mathrm{a}$ & $76.7 \pm 0.57 \mathrm{a}$ & $80.5 \pm 1.03 \mathrm{a}$ & $53.3 \pm 0.57 \mathrm{~b}$ & $31.4 \pm 1.01 \mathrm{c}$ & $20.0 \pm 0.03 \mathrm{c}$ \\
Diaeretiella & $10^{4}$ & $73.3 \pm 1.15 \mathrm{a}$ & $60.0 \pm 0.01 \mathrm{~b}$ & $66.7 \pm 1.15 \mathrm{a}$ & $36.7 \pm 0.57 \mathrm{c}$ & $20.0 \pm 0.03 \mathrm{c}$ & $13.3 \pm 1.01 \mathrm{c}$ \\
rapae & $10^{8}$ & $99.9 \pm 0.01 \mathrm{a}$ & $96.5 \pm 0.57 \mathrm{a}$ & $98.6 \pm 0.33 \mathrm{a}$ & $76.7 \pm 0.57 \mathrm{~b}$ & $56.6 \pm 0.66 \mathrm{c}$ & $7.2 \pm 0.01 \mathrm{~d}$ \\
& $10^{7}$ & $91.2 \pm 1.73 \mathrm{a}$ & $86.4 \pm 0.66 \mathrm{a}$ & $90.2 \pm 0.57 \mathrm{a}$ & $60.0 \pm 0.74 \mathrm{~b}$ & $41.6 \pm 0.08 \mathrm{~b}$ & $12.3 \pm 0.03 \mathrm{c}$ \\
& $10^{6}$ & $80.3 \pm 0.57 \mathrm{a}$ & $76.7 \pm 0.33 \mathrm{a}$ & $73.3 \pm 1.30 \mathrm{a}$ & $43.8 \pm 0.01 \mathrm{~b}$ & $33.1 \pm 0.07 \mathrm{~b}$ & $6.7 \pm 0.33 \mathrm{c}$ \\
& $10^{5}$ & $63.3 \pm 0.66 \mathrm{a}$ & $56.7 \pm 0.33 \mathrm{a}$ & $53.3 \pm 0.23 \mathrm{a}$ & $46.7 \pm 0.33 \mathrm{~b}$ & $26.7 \pm 0.33 \mathrm{c}$ & $20.1 \pm 0.03 \mathrm{c}$ \\
& $10^{4}$ & $56.7 \pm 0.33 \mathrm{a}$ & $51.0 \pm 0.57 \mathrm{a}$ & $44.1 \pm 0.36 \mathrm{a}$ & $26.7 \pm 0.23 \mathrm{~b}$ & $10.6 \pm 0.03 \mathrm{bc}$ & $6.7 \pm 1.01 \mathrm{c}$ \\
\hline
\end{tabular}

Note. Means followed by the same superscript letter(s), within the same rows are insignificantly different $(P \leq 0.05)$ according to Tukey HSD test; SE - standard error.

with $\left(\mathrm{F}_{11,24}=95.68, P=0.001\right),\left(\mathrm{F}_{11,24}=75.29, P=0.001\right)$ and $\left(\mathrm{F}_{11,24}=89.36, P=0.001\right)$, $\left(\mathrm{F}_{11,24}=156.09, P=\right.$ $0.001)$, respectively. According to the results, the highest mortality of $B$. brassicae after 48 hours was by $\mathrm{LC}_{25}$ (imidacloprid $+A$. sclerotigenum), $\mathrm{LC}_{25}$ (imidacloprid + B. bassiana), $\mathrm{LC}_{25}$ (imidacloprid + L. muscarium) and imidacloprid, and after 72 hours by $\mathrm{LC}_{25}$ (imidacloprid + A. sclerotigenum), $\mathrm{LC}_{25}$ (imidacloprid $+B$. bassiana), $\mathrm{LC}_{25}$ (imidacloprid $+P$. variotii), $\mathrm{LC}_{25}$ (imidacloprid + Simplicillium sp.), $\mathrm{LC}_{25}$ (imidacloprid + L. muscarium) and imidacloprid (Fig. 1). The highest mortality of $D$. rapae after 48 hours was by $\mathrm{LC}_{25}$ (imidacloprid + A. sclerotigenum), $\mathrm{LC}_{25}$ (imidacloprid $+B$. bassiana), $\mathrm{LC}_{25}$ (imidacloprid + L. muscarium) and imidacloprid, and after 72 hours by $\mathrm{LC}_{25}$ (imidacloprid + A. sclerotigenum), $\mathrm{LC}_{25}$ (imidacloprid + B. bassiana), $\mathrm{LC}_{25}$ (imidacloprid + L. muscarium) and imidacloprid (Fig. 2).

The percentage of $D$. rapae emergence. The effect of different treatments on the percentages of D. rapae emergence showed that there were significant

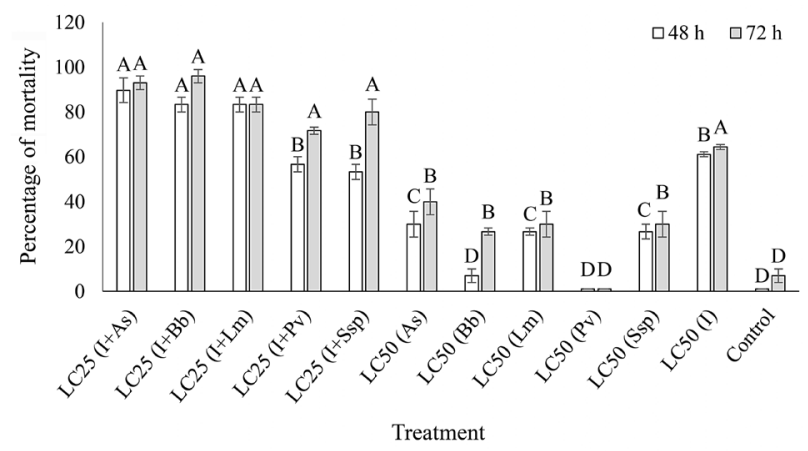

Note. The columns with the same letters indicate nonsignificant differences between the treatments; I - imidacloprid, As - Acremonium sclerotigenum, Bb-Beauveria bassiana, Lm - Lecanicillium muscarium, Pv-Paecilomyces variotii, SspSimplicillium $\mathrm{sp.}$

Figure 1. Mean $( \pm \mathrm{SE})$ efficacy (\%) of treatments against adult of Brevicoryne brassicae after 48 and 72 hours in laboratory conditions differences between the treatment with $\left(\mathrm{F}_{11,24}=65.129\right.$, $P=0.001)$. The result showed that the percentage of parasitoid emergence in $P$. variotii and Simplicillium sp. treatments was higher than in the other treatments (Fig. 3)

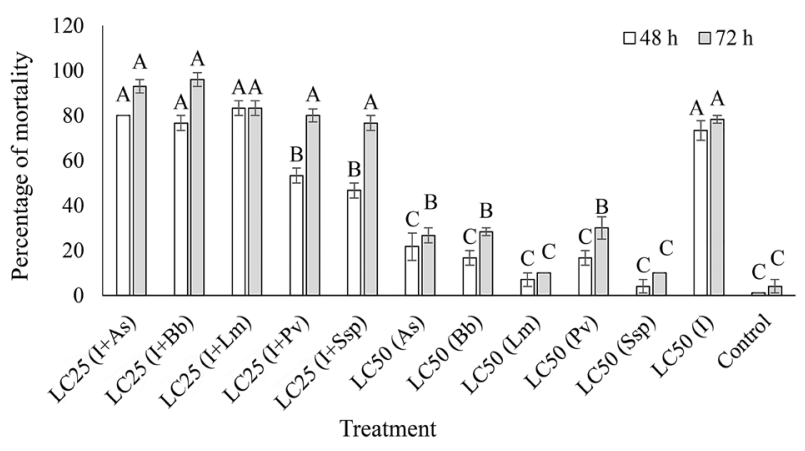

Note. The columns with the same letters indicate non-significant differences between treatments; explanation under Figure 1.

Figure 2. Mean ( \pm SE) efficacy (\%) of treatments against adult of Diaeretiella rapae after 48 and 72 hours in laboratory conditions

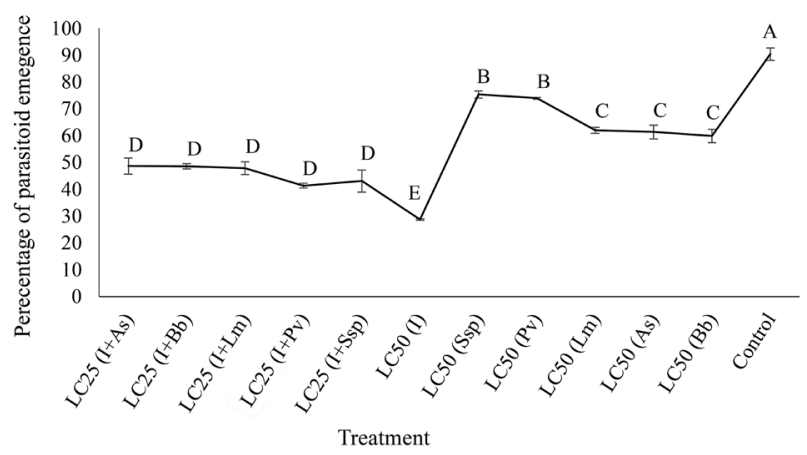

Note. The same letters indicate non-significant differences between the treatments; explanation under Figure 1.

Figure 3. Mean ( \pm SE) emergence (\%) of Diaeretiella rapae on treated Brevicoryne brassicae 


\section{Discussion}

The role of entomopathogenic fungi as prominent biological control agents has shown that the fungal isolates affected the aphids and parasitoids (Kim, 2007; Roditakis et al., 2008). The results of our experiment showed that, based on $\mathrm{LC}_{50}$ and $\mathrm{LT}_{50}$ values, L. muscarium was an effective isolate against aphid and parasitoid. Combinations of fungal isolates with imidacloprid, $\mathrm{LC}_{25}$ (imidacloprid $+A$. sclerotigenum), $\mathrm{LC}_{25}$ (imidacloprid + B. bassiana) and $\mathrm{LC}_{25}$ (imidacloprid + L. muscarium) were more effective against aphid. All fungal isolates showed virulence against $B$. brassicae after 3-7 days of treatments. The fungal isolate Lecanicillium lecanii was the most virulent among the fungal isolates tested, followed by Metarhizium anisopliae, Paecilomyces fumosoroseus and $M$. anisopliae against the adult aphids (Asi et al., 2009), and in our study, A. sclerotigenum, $B$. bassiana and $P$. variotii were virulent among isolates. The results indicated that $M$. anisopliae had larvicidal effect on Aedes albopictus with $1.09 \times 10^{5}$ conidia $\mathrm{ml}^{-1}$, while it took $45.41 \mathrm{~h}$ to kill $50 \%$ of the tested population; in our research $50 \%$ mortality by $A$. sclerotigenum at $10^{8}$ concentration was observed after 0.28 days $(6.72 \mathrm{~h})$.

In current study, $\mathrm{LC}_{50}$ and $\mathrm{LT}_{50}$ values of B. bassiana were $3.7 \times 10^{3}$ conidia $\mathrm{ml}^{-1}$ and 1.92 day at $10^{8}$ concentration - similar to Saruhan et al. (2015), who tested B. bassiana on different aphids such as B. brassicae and Aphis fabae. It was found to be effective at all concentrations, i.e. $10^{6}, 10^{7}$ and $10^{8}$ conidia $\mathrm{ml}^{-1}$, against all aphid species, but the uppermost $\left(10^{8}\right.$ conidia $\left.\mathrm{ml}^{-1}\right)$ concentration provided maximum control within a short period of time. Taking long time to kill $50 \%$ population by fungal isolates compared to synthetic insecticides is the only drawback for the application of entomopathogenic fungi, but these biopesticides are safe for use (Bilal et al., 2012). The fitness of the parasitoids that emerge from insecticide treated hosts is very important (Bayram et al., 2010; Saber, 2011).

The parasitoid Aphidius colemani developed normally (approximately 90\% adult emergence) when its cotton aphid (Aphis gossypii) host was treated with $V$. lecanii conidia 5 or 7 days after parasitization. Fungus exposure 1 day before or up to 3 days after parasitization reduced $A$. colemani emergence from $0 \%$ to $10 \%$ (Kim et al., 2005). In our experiment, 7 days after application of fungal isolates, the emergence percentage of D. rapae decreased from $30 \%$ to $70 \%$ (Fig. 3). The $\mathrm{LT}_{50}$ values showed L. muscarium to be the most effective entomopathogenic fungus against Aphis fabae at both $20^{\circ} \mathrm{C}$ and $25^{\circ} \mathrm{C}$ temperatures (1.77 and 1.93 day), followed by Simplicillium lamellicola (2.12 and 1.96 day) and $V$. lecanii (2.33 and 2.03 day). Also in this research, $\mathrm{LT}_{50}$ values of $A$. sclerotigenum, $B$. bassiana, $P$. variotii, Simplicillium sp. and $L$. muscarium on adult of $B$. brassicae was $0.26,1.92,7.40,2.92$ and 1.32 days, respectively.

Various authors have reported different results in the combined action of fungal strains and chemical insecticides. For example, the combination on B. bassiana-diflubenzuron was more effective than the pairing of $B$. bassiana with other chemical agents in field applications against grasshoppers in the United States (Foster et al., 1996) and in populations of grasshoppers in Mali treated with a mixture of $B$. bassiana and diflubenzuron (Delgado et al., 1999). It was observed that the decrease in the number of insects continued until the end of the monitoring (Bisadze et al., 2013).

The present study showed that $\mathrm{LC}_{50}$ concentration of imidacloprid severely decreased the emergence of parasitoids, but when $\mathrm{LC}_{25}$ of imidacloprid plus $\mathrm{LC}_{25}$ of each fungal isolate had been used, parasitoid emergence decreased (Fig. 3). Similarly, reduction in emergence rate was reported in Encarsia inaron after treatment with imidacloprid (Sohrabi et al., 2012). Imidacloprid also led to significant mortality of Eretmocerus eremicus, E. mundus and Encarsia formosa (Sugiyama et al., 2011) and E. inaron adult (Sohrabi et al., 2012).

According to the above results of current research, imidacloprid was highly toxic to adult $D$. rapae with an $\mathrm{LC}_{50}$ value of $12.64 \mathrm{ppm}$. In our experiment, imidacloprid had harmful effects on both cabbage aphid and its parasitoid with $64.42 \%$ and $78.33 \%$ mortality, respectively. Similar result was observed in research of Towfiq et al. (2010), who reported that pirimiphosmethyl, thiamethoxam, malathion and thiacloprid were highly toxic to parasitoids and cabbage aphids. According to the manufacturer's label, a single application of B. bassiana (insecticide Vertalec) can effectively control A. gossypii for up to 5 days in greenhouse environments (Kim, 2007).

In our experiment, B. brassicae was controlled by $B$. bassiana after 7 days. Among the entomopathogenic fungi, L. muscarium, A. sclerotigenum and B. bassiana had higher mortality rate of $B$. brassicae and D. rapae in combination with imidacloprid, but fungal isolates had the lower effect on D. rapae in alone form. Results of current experiment showed that fungal isolates can be used for control of B. brassicae in IPM program.

\section{Conclusion}

There is a general misperception that entomopathogenic fungi are not harmful to natural enemies. However, based on the information in this article, it is necessary to exercise caution in applying entomopathogenic fungi alone and in combination with a pesticide for conservation and augmentation of natural enemies.

Hence, further studies should be conducted under more realistic environments (e.g., greenhouse and field) on simultaneous application of pesticide, entomopathogenic fungi and parasitoids in management this pest.

Received 23092019 Accepted 17012020

\section{References}

1. AbbottW. S. 1925. A method of computing the effectiveness of an insecticide. Journal of Economic Entomology, 18: 265-267. https://doi.org/10.1093/jee/18.2.265a

2. Al-Olayan E. M. 2013. Evaluation of pathogenicity of certain mitosporic ascomycete fungi to the house fly, Musca domestica L. (Diptera: Muscidae). Journal of Saudi Chemical Society, 17 (1): 97-100. https://doi.org/10.1016/j.jscs.2011.11.019

3. Asi M. R., Bashir M. H., Afzal M., Imran S. 2009. Effect of conidial concentration of entomopathogenic fungi on mortality of cabbage aphid, Brevicoryne brassicae L. Pakistan Journal of Life and Social Sciences, 2: 175-180. Bayram A., Salerno G., Onofri A., Conti E. 2010. Sub- 
lethal effects of two pyrethroids on biological parameters and behavioral responses to host cues in the egg parasitoid Telenomus busseolae. Biological Control, 53 (2): 153-160. https://doi.org/10.1016/j.biocontrol.2009.09.012

4. Bayramzadeh N., Mehrkhou F., Pourmirza A. A., Mahmoudian M. 2019. Fumigant toxicity of two nanocapsulated essential oils with sublethal rate of phosphine against three stored product pests. Journal of Agriculture and Science Technology, 21 (4): 857-872.

5. Bilal H., Hassan S. A., Khan I. A. 2012. Isolation and efficacy of entomopathogenic fungus (Metarhizium anisopliae) for the control of Aedes albopictus Skuse larvae: suspected dengue vector in Pakistan. Asian Pacific Journal of Tropical Biomedicine, 2 (4): 298-300. https://doi.org/10.1016/S2221-1691(12)60026-4

6. Bisadze N., Jaronski S., Khasdan V., Abashidze E., Abashidze M., Latchininsky A., Samadashvili D., Sokhadze I., Rippa M., Ishaaya I., Horowitz A. R. 2013. Joint action of Beauveria bassiana and the insect growth regulators diflubenzuron and novaluron, on the migratory locust, Locusta migratoria. Journal of Pest Science, 86 (2): 293-300. https://doi.org/10.1007/s10340-012-0476-4

7. Brennan E. B. 2016. Agronomy of strip intercropping broccoli with alyssum for biological control of aphids. Biological Control, 97: 109-119.

https://doi.org/10.1016/j.biocontrol.2016.02.015

8. Collier R. H., Finch S. 2017. IPM case studies: Brassicae. Emden van H., Harrington R. (eds). Aphids as crop pests, p. 549-559. https://doi.org/10.1079/9780851998190.0549

9. Delgado F. X., Britton J. H., Onsager J. A., Swearingen W. 1999. Field assessment of Beauveria bassiana (Balsamo) Vuillemin and potential synergism with Diflubenzuron for control of Savanna grasshoppers complex (Orthoptera) in Mali. Journal of Invertebrate Pathology, 73 (1): 34-39. https://doi.org/10.1006/jipa.1998.4804

10. Fadayivata S., Moravvej G. H., Karimi J. 2014. Pathogenicity of the fungus Lecanicillium longisporum against Sipha maydis and Metopolophium dirhodum in laboratory conditions. Journal of Plant Protection Research, 54 (1): 67-73. https://doi.org/10.2478/jppr-2014-0010

11. Foster R. N., Reuter K. C., Black L., Britton J. 1996. Evaluation of the fungus Beauveria bassiana with selected insecticide stressors for control of unconfined rangeland grasshoppers. Arthropod Management Tests, 21 (1): 280. https://doi.org/10.1093/amt/21.1.280

12. Güçlü S., Ak K., Eken C., Akyol H., Sekban R., Beytut B., Yildirim R. 2010. Pathogenicity of Lecanicillium muscarium against Ricania simulans. Bulletin of Insectology, 63 (2): 243-24.

13. Gurulingappa P., McGee P., Sword G. A. 2011. In vitro and in planta compatibility of insecticides and the endophytic entomopathogen, Lecanicillium lecanii. Mycopathology, 172 (2): 161-168. https://doi.org/10.1007/s11046-011-9410-1

14. Jones V. P., Unruh T. R., Horton D. R., Mills N. J., Brunner J. F., Beers E. H., Shearer P. W. 2009. Tree fruit IPM programs in the western United States: the challenge of enhancing biological control through intensive management. Pest Management Science, 65 (12): 1305 1310. https://doi.org/10.1002/ps.1839

15. Kim J. J. 2007. Influence of Lecanicillium attenuatum on the development and reproduction of the cotton aphid, Aphis gossypii. BioControl, 52: 789-799. https://doi.org/10.1007/s10526-006-9050-4

16. Kim J. J., Kim K. C., Roberts D. W. 2005. Impact of the entomopathogenic fungus Verticillium lecanii on development of an aphid parasitoid, Aphidius colemani. Journal of Invertebrate Pathology, 88 (3): 254-256. https://doi.org/10.1016/j.jip.2005.01.004
17. Kim D. S., Brooks D. J., Riedl H. 2006. Lethal and sublethal effects of abamectin, spinosad, methoxyfenozide and acetamiprid on the predaceous plant bug Deraeocoris brevis in the laboratory. BioControl, 51: 465-484. https://doi.org/10.1007/s10526-005-1028-0

18. Lazreg F., Huang Z., Ali S., Ren S. 2009. Effect of Lecanicillium muscarium on Eretmocerus sp. nr. furuhashii (Hym.: Aphelinidae), a parasitoid of Bemisia tabaci (Hem.: Aleyrodidae). Journal of Pest Science, 82: 27-32. https://doi.org/10.1007/s10340-008-0215-z

19. Loureiro E. S., Moino Jr. A. 2006. Pathogenicity of hyphomycete fungi to aphids, Aphis gossypii Glover and Myzus persicae (Sulzer) (Hemiptera: Aphididae). Neotropical Entomology, 35 (5): 660-665 (in Portuguese). https://doi.org/10.1590/S1519-566X2006000500014

20. Neuville S., Le Ralec A., Outreman Y., Jaloux B. 2015. The delay in arrival of the parasitoid Diaeretiella rapae influences the efficiency of cabbage aphid biological control. BioControl, 61: 115-126. https://doi.org/10.1007/s10526-015-9702-3

21. Pereira A. J., Cardoso I. M., Araujo H. D., Santana F. C., Carneiro A. P. s., Coelho S. P., Pereira F. J. 2018. Control of Brevicoryne brassicae (Hemiptera: Aphididae) with extracts of Agave americana var. marginata Trel. in Brassica oleracea crops. Annals of Applied Biology, 174 (1): 14-19. https://doi.org/10.1111/aab.12471

22. Rashki M., Kharazi-Pakdel A., Allahyari H., van Alphen J. J. M. 2009. Interactions among the entomopathogenic fungus, Beauveria bassiana (Ascomycota: Hypocreales), the parasitoid, Aphidius matricariae (Hymenoptera: Braconidae) and its host, Myzus persicae (Homoptera: Aphididae). Biological Control, 50 (3): 324-328. https://doi.org/10.1016/j.biocontrol.2009.04.016

23. Rashki M., Talepour F., Shirvani A. 2015. Sub-lethal effect of combination of Metarhizium anisopliae and imidacloprid on life table of Myzus persicae (Hem.: Aphididae). Journal of Crop Protection, 4 (4): 577-587.

24. Roditakis E., Couzin I. D., Franks N. R., Charnley A. K. 2008. Effects of Lecanicillium longisporum infection on the behaviour of the green peach aphid Myzus persicae. Journal of Insect Physiology, 54 (1): 128-136. https://doi.org/10.1016/j.jinsphys.2007.08.008

25. Roessink I., Merga L. B., Zweers H. J., Van Den Brink P. J. 2013. The neonicotinoid imidacloprid shows high chronic toxicity to mayfly nymphs. Environmental Toxicology and Chemistry, 32 (5): 1096-1100. https://doi.org/10.1002/etc. 2201

26. Saber M. 2011. Acute and population level toxicity of imidacloprid and fenpyroximate on an important egg parasitoid, Trichogramma cacoeciae (Hymenoptera: Trichogrammatidae). Ecotoxicology, 20 (6): 1476-1484. https://doi.org/10.1007/s10646-011-0704-3

27. Sain S. K., Monga D. Kumar R., Nagrale D. T., Hiremani N. S., Kranthi S. 2019. Compatibility of entomopathogenic fungi with insecticides and their efficacy for IPM of Bemisia tabaci in cotton. Journal of Pesticide Science, 44 (2): 97-105. https://doi.org/10.1584/jpestics.D18-067

28. Saleh A. A. A., Desuky W. M., Hashem H. H., Gatwarry W. G. 2009. Evaluating the role of Diaeretiella rapae (M'Intosh) (Hymenoptera: Aphidiidae) parasitizing the cabbage aphid, Brevicoryne brassicae L. (Homoptera: Aphididae) at Sharkia Governorate, Egypt. Egyptian Journal of Biological Pest Control, 19 (2): 151-155.

29. Sandhu S. S., Sharma K. A., Beniwal V., Goel G., Batra P., Kumar A., Jaglan S., Sharma A. K., Malhotra S. 2012. Myco-biocontrol of insect pests: factors involved, mechanism, and regulation. Journal of Pathogens, 2012: 126819. https://doi.org/10.1155/2012/126819 
30. Saruhan I., Ismail Erper I., Tuncer C., Izzet Akca I. 2015. Efficiency of some entomopathogenic fungi as biocontrol agents Aphis fabae Scopoli (Hem.: Aphodidae). Pakistan Journal of Agricultural Sciences, 52 (2): 273-278.

31. Seiedy M., Heydari S., Tork M. 2015. Orientation of Hippodamia variegate (Coleoptera: Coccinellidae) to healthy and Beauveria bassiana-infected Aphis fabae (Hemiptera: Aphididae) in an olfactometer system. Turkish Journal of Zoology, 39: 53-58.

https://doi.org/10.3906/zoo-1310-18

32. Shah P. A., Pell J. K. 2003. Entomopathogenic fungi as biological control agents. Applied Microbiology and Biotechnology, 61: 413-423.

https://doi.org/10.1007/s00253-003-1240-8

33. Singh R., Singh G. 2015. Systematics, distribution and host range of Diaeretiella rapae (McIntosh) (Hymenoptera: Braconidae, Aphidiinae). International Journal of Research Studies in Biosciences, 3 (1): 1-36.

34. Sohrabi F., Shishehbor P., Saber M., Mosaddegh M. S. 2012. Lethal and sublethal effects of buprofezin and imidacloprid on the whitefly parasitoid Encarsia inaron (Hymenoptera: Aphelinidae). Crop Protection, 32: 83-89. https://doi.org/10.1016/j.cropro.2011.10.005
35. Song Y. Q., Sun H. Z., Du J., Wang X. D., Cheng Z. J. 2017. Evaluation of Aphis glycines as an alternative host for supporting Aphelinus albipodus against Myzus persicae on Capsicum annuum cv. Ox Horn and Hejiao 13. Neotropical Entomology, 46 (2): 193-202. https://doi.org/10.1007/s13744-016-0456-0

36. Sugiyama K., Katayama H., Saito T. 2011. Effect of insecticides on the mortalities of three whitefly parasitoid species, Eretmocerus mundus, Eretmocerus eremicus and Encarsia formosa (Hym.: Aphelinidae). Applied Entomology and Zoology, 46 (2): 1-7. https://doi.org/10.1007/s13355-011-0044-z

37. Towfiq M., Al A., Ateyyat M. A., Abussamin B. M. 2010. Toxicity of certain insecticides to the parasitoid Diaeretiella rapae (Mcintosh) (Hym.: Aphidiidae) and its host, the cabbage aphid Brevicoryne brassicae L. (Hom.: Aphididae). Australian Journal of Basic and Applied Sciences, 4 (6): 994-1000.

\title{
ISSN 1392-3196 / e-ISSN 2335-8947
}

Zemdirbyste-Agriculture, vol. 107, No. 3 (2020), p. 255-262

DOI 10.13080/z-a.2020.107.033

\section{Grybinių izoliatų ir imidakloprido poveikis kopūstiniam amarui Brevicoryne brassicae ir jo parazitoidui Diaeretiella rapae}

\author{
S. Akbari, Sh. Mirfakhraie, Sh. Aramideh, M. H. Safaralizadeh \\ Urmia universiteto Žemès ūkio mokslų fakultetas, Iranas
}

\section{Santrauka}

Diaeretiella rapae (M’Intosh) yra pirminis kopūstinio amaro Brevicoryne brassicae (L.) parazitas; abi rūšys yra plačiai paplitusios visame pasaulyje. Tyrimo metu buvo ịvertintas imidakloprido ir penkių entomopatogeninių grybų: Acremonium sclerotigenum, Beauveria bassiana, Paecilomyces variotii, Simplicillium sp. ir Lecanicillium muscarium, efektyvumas kopūstiniam amarui ir jo parazitoidui. Siekiant ịvertinti visų izoliatų ir imidakloprido $\mathrm{LC}_{50}$ (mirtinos koncentracijos) ir $\mathrm{LT}_{50}$ (mirtinos laiko trukmès) vertes $B$. brassicae ir $D$. rapae suaugèliams, šie mirtingumo duomenys analizuoti taikant Probit analizę. A. sclerotigenum, B. bassiana, P. variotii, Simplicillium sp., L. muscarium

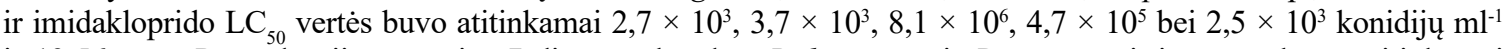
ir 13,56 ppm. Po apdorojimo praejus 7 dienoms bendras $B$. brassicae ir D. rapae mirtingumas buvo atitinkamai 98,56 ir 99,99 \% panaudojus didelę L. muscarium koncentraciją $\left(10^{8}\right.$ konidijų $\left.\mathrm{ml}^{-1}\right)$. Didžiausias $B$. brassicae ir D. rapae mirtingumas po 48 ir 72 valandų buvo nustatytas paveikus šių derinių subletalinèmis kombinacijomis: $\mathrm{LC}_{25}$ (imidaklopridas + A. sclerotigenum), $\mathrm{LC}_{25}$ (imidaklopridas + B. bassiana) bei $\mathrm{LC}_{25}$ (imidaklopridas + L. muscarium) ir $\mathrm{LC}_{25}$ (imidaklopridas + A. sclerotigenum), $\mathrm{LC}_{25}$ (imidaklopridas + B. bassiana), $\mathrm{LC}_{25}$ (imidaklopridas + P. variotii), $\mathrm{LC}_{25}$ (imidaklopridas + Simplicillium sp.) bei $\mathrm{LC}_{25}$ (imidaklopridas + L. muscarium). B. brassicae ir D. rapae paveikus $10^{8}$ konidiju $\mathrm{ml}^{-1}$ koncentracija, LT50 vertès buvo 0,26 bei 2,16 dienos, panaudojus $A$. sclerotigenum izoliatą, ir 7,4 bei 6,22 dienos, panaudojus $P$. variotii izoliatą. Nustatyta, kad imidaklopridas turèjo žalingą poveikị ir kenkejjui, ir parazitoidui; grybiniai izoliatai parazitoidui buvo saugesni už imidaklopridą.

Tyrimo rezultatai parodè, kad taikant integruotą B. brassicae kontrolę, entomopatogeniniai grybai gali būti efektyviai naudojami atskirai ir mišinyje su subletaline $\left(\mathrm{LC}_{25}=9,23 \mathrm{ppm}\right)$ imidakloprido koncentracija.

Reikšminiai žodžiai: biologinè kontrolè, entomopatogeniniai grybai, parazitoidas, patogeniškumas, subletalinis. 